Original paper

\title{
Comparison of hepatic and splenic stiffness in chronic hepatitis $C$ infection with and without schistosomal infection; correlation with hepatic histopathological changes
}

\author{
Ahmed Al Gohary, Amany Elyamany, Dina Abdalla, Ahmed Abolfadl \\ Faculty of Medicine, Alexandria University, Alexandria, Egypt
}

\begin{abstract}
Aim of the study: To assess the degree of liver and spleen stiffness in chronic hepatitis $\mathrm{C}$ virus (HCV) patients co-infected with schistosomiasis, and chronic HCV mono-infected patients.

Material and methods: The present study was conducted on 50 Egyptian chronic HCV patients, categorized into two groups: group A (25 patients with chronic HCV mono-infection) and group B (25 patients with chronic $\mathrm{HCV}$ and schistosomiasis coinfection). Also, 25 age- and sex-matched healthy subjects with no evidence of liver disease were included in the study as a control group. Stage of fibrosis was assessed invasively by histopathological examination of liver biopsies (patients only) and non-invasively by acoustic radiation force impulse imaging (ARFI) (all subjects).

Results: ARFI of liver (ARFI L) and ARFI of spleen (ARFI S) in group A patients who had significant fibrosis were $1.82 \pm 0.24$ and $2.72 \pm 0.26$, respectively, while in group B, ARFI L and ARFI S in patients with significant fibrosis was $1.99 \pm 0.53$ and $3.10 \pm 0.57$, respectively.

Conclusions: The current study demonstrated insignificantly higher values of liver stiffness and significantly higher values of spleen stiffness assessed by ARFI in patients co-infected with HCV and schistosomiasis than mono-infected with HCV.
\end{abstract}

Key words: elasticity, stiffness, hepatitis C virus, schistosomiasis.

Address for correspondence:

Prof. Amany Elyamany, Faculty of Medicine, Champollion Street, El-Khartoum Square, El Azareeta Medical Campus, Alexandria University, 21131, Alexandria, Egypt, e-mail: amanyyamany@yahoo.com

\section{Introduction}

The seroprevalence of hepatitis $\mathrm{C}$ virus $(\mathrm{HCV})$ in Egypt was reported to be $10 \%[1,2]$. Schistosomiasis, a common condition in Egypt, is characterized by the formation of a granulomatous inflammatory reaction, which leads to periportal fibrosis formation [3]. Management of patients with $\mathrm{HCV}$ is contingent on the intensity of fibrosis, which is established by using acoustic radiation force impulse imaging (ARFI), a non-invasive technique. It utilizes focused acoustic beams to intra-convert acoustic compressional waves to shear waves [1].
The grade of the stiffness of the liver and spleen in chronic HCV patients (with and without schistosomiasis) was assessed and correlated with the histopathological changes.

\section{Material and methods}

This is a pilot study that was conducted on 50 Egyptian chronic HCV patients classified into two groups. Group A consisted of 25 patients with chronic HCV while group B comprised 25 patients with chronic $\mathrm{HCV}$ co-infected with schistosomiasis. 
All patients' sera were positive for circulating HCV antibodies and HCV RNA and all had histopathological findings consistent with chronic hepatitis ( $\mathrm{CHC}$ ). Patients in group B tested positive for human serum anti-schistosomal antibody and exhibited peri-portal thickening (PPT) in the ultrasonographic examination. As a control group, 25 healthy, age- and sexmatched subjects with no evidence of liver disease were recruited to establish normal ARFI elastography of the liver and spleen.

The study was conducted per the provisions of the Declaration of Helsinki and Good Clinical Practice guidelines. Institutional Review Board approval was obtained prior to study initiation. Informed consent was obtained from all study participants. Exclusion criteria included coinfection with hepatitis B virus, history of alcohol consumption, known causes of chronic liver disease, chronic disorders such as cardiac diseases, diabetes mellitus, hyperlipidaemia, malignancies, and connective tissue, infectious or inflammatory disorders.

Screening procedures consisted of full medical history, physical examination, laboratory investigations including complete blood count (CBC), serum aspartate aminotransferase (AST), alanine aminotransferase (ALT), serum albumin, serum bilirubin, and serum $\gamma$-glutamyl transpeptidase (GGT) and prothrombin time (PT), HCV antibody, hepatitis B surface antigen and hepatitis B core antibody using ELISA, and serum anti-schistosomal antibodies using the enzyme-linked immunosorbent assay (ELISA). PCR assay was used to determine the HCV RNA level utilizing the TaqMan technology from Applied Biosystems and the automated extractor QIA cube (ABI PRISM 7500 sequence detection system).

Abdominal ultrasound was performed in all participants to assess the liver and spleen size and determine PPT. All subjects underwent ARFI elastography

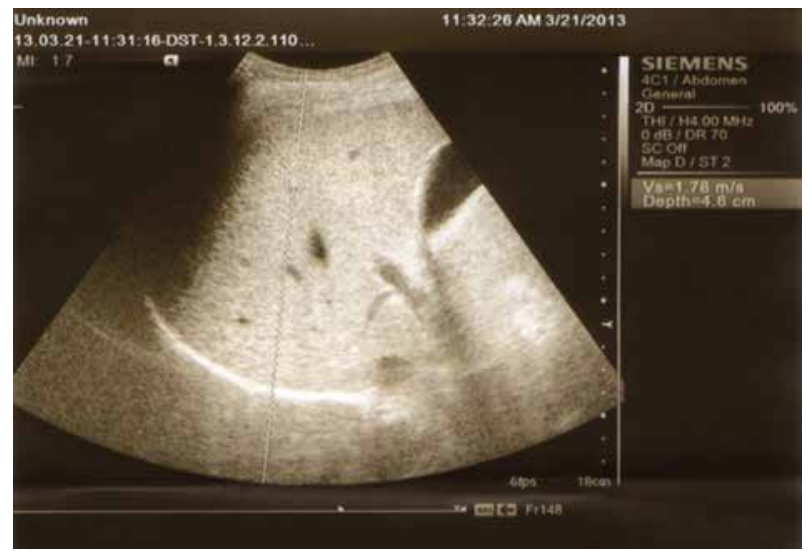

Fig. 1. ARFI measurement of the liver and spleen using a Siemens Acuson S3000 Virtual Touch ultrasound system (Siemens AG, Erlangen, Germany) employing a $4 \mathrm{CI}$ transducer. The shear waves generated by the acoustic pulse propagates into the tissue and is directed to the side of a region of interest (ROI); their speed is measured in meters per second. The examination was carried out by the intercostal approach in the right liver lobe, $1-2 \mathrm{~cm}$ under the liver capsule; while the patient was fasting, in the supine position and with the right arm fully abducted. The patient was asked to hold the breath for a moment. The mean of 8-10 valid measurements was determined (Fig. 1). All participants were assessed by the same operator who conducted the sonographic and ARFI screening to avoid inter-observer variability.

\section{Histopathological evaluation}

Using a semi-automatic true-cut needle (16 G), both groups (not the control group) were subjected to core liver biopsies on the same day following ARFI elastography. Specimens were fixed in a $10 \%$ solution of formalin and embedded in paraffin. Tissue sections were subsequently stained with haematoxylin-eosin and trichrome stains for histopathological diagnosis, assessment of the degree of necro-inflammatory activity (grading) and extent of fibrosis (staging) according to the METAVIR scoring system. Fibrosis was staged on a $0-4$ scale: F0 - no fibrosis, F1 - portal fibrosis without septa, F2 portal fibrosis with a few septa extending into lobules, F3 - numerous septa extending to adjacent portal tracts or terminal hepatic venules, and F4 - cirrhosis.

\section{Statistical analysis}

The collected data were analysed using SPSS version 11 software. Results were expressed as percentages, means and standard deviations (SD). Statistical

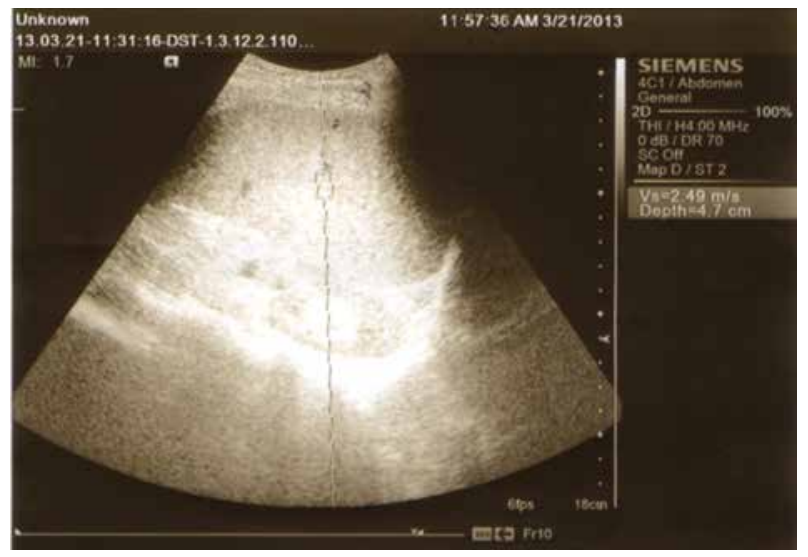


tests used in the present study were the following: 1) Student's $t$ test as a test of significance for comparison between two arithmetic means; 2) the Mann-Whitney $U$-test for comparison between two means of non-normally distributed variables; 3) the Pearson correlation coefficient to evaluate the strength of correlation ( $r$ value) between continuous quantitative variables; 4) the Spearman rho correlation coefficient to detect the strength of correlation ( $r$ value) between discontinuous quantitative variables. The $5 \%$ level was chosen as the cut-off level of significance.

\section{Results}

The characteristics of the studied patients are presented in Table 1. Histopathological features were ob- served in all liver specimens including the presence of lymphoid aggregation in portal tracts, variable degrees of steatosis and bile duct proliferation as shown in Figures 2 and 3. According to the METAVIR scoring system in regards to the fibrosis stage, $12(48 \%)$ patients in group A showed F1, 12 (48\%) patients showed F2 and only one (4\%) patient showed F3. Patients in group B were found to have a significant increase in the fibrosis stage, where six (24\%) showed F1, 12 (48\%) showed F2 and seven (28\%) patients showed F3.

Table 2 represents the results of liver and spleen stiffness assessed by ARFI (ARFI L, ARFI S) in the studied subjects. ARFI L measured in group $B$ patients has insignificantly higher values than that measured in patients with group A, while ARFI S measured in group B patients has significantly higher values than

Table 1. Characteristic features of the studied groups

\begin{tabular}{|c|c|c|c|c|}
\hline Variables & $\begin{array}{l}\text { Group A } \\
(n=25)\end{array}$ & $\begin{array}{l}\text { Group B } \\
(n=25)\end{array}$ & $\begin{array}{l}\text { Control } \\
(n=25)\end{array}$ & Test of Sig. \\
\hline \multicolumn{5}{|l|}{ Gender } \\
\hline Male & $15(60 \%)$ & $22(88 \%)$ & $14(56 \%)$ & $x^{2} p=0.030^{*}$ \\
\hline Female & $10(40 \%)$ & $3(12 \%)$ & $11(44 \%)$ & \\
\hline Age (years) & $45.08 \pm 7.29$ & $42.32 \pm 7.15$ & $33.0 \pm 5.42$ & ${ }^{\mathrm{kW}} p<0.001^{*}$ \\
\hline $\mathrm{Hb}$ & $14.29 \pm 1.38$ & $15.17 \pm 1.83$ & $14.06 \pm 1.17$ & $\mathrm{~F} p=0.026^{*}$ \\
\hline WBCs & $6.64 \pm 1.56$ & $7.77 \pm 1.59$ & $6.31 \pm 1.39$ & $\mathrm{~F} p=0.003^{*}$ \\
\hline Platelets & $213.28 \pm 72.46$ & $193.56 \pm 72.31$ & $321.0 \pm 78.17$ & ${ }^{\mathrm{KW}} p<0.001^{*}$ \\
\hline AST & $27.64 \pm 23.05$ & $25.96 \pm 20.21$ & $20.12 \pm 3.26$ & $\mathrm{~F} p=0.299$ \\
\hline ALT & $30.28 \pm 26.91$ & $31.24 \pm 28.24$ & $20.0 \pm 4.56$ & ${ }^{\mathrm{kW}} p=0.728$ \\
\hline Albumin & $4.33 \pm 0.45$ & $4.01 \pm 0.70$ & $4.6 \pm 0.48$ & ${ }^{\mathrm{kW}} p=0.10^{*}$ \\
\hline Total bilirubin & $0.83 \pm 0.17$ & $0.88 \pm 0.36$ & $0.66 \pm 0.16$ & ${ }^{\mathrm{kW}} p=0.003^{*}$ \\
\hline PA & $91.64 \pm 9.97$ & $94.08 \pm 7.57$ & $77.25 \pm 34.14$ & ${ }^{\mathrm{kW}} p=0.062$ \\
\hline $\mathrm{RNA} \times 10^{3}(\mathrm{IU} / \mathrm{ml})$ & $812.66 \pm 1439.04$ & $679.44 \pm 838.66$ & & 0.741 \\
\hline \multicolumn{5}{|c|}{ Grade of inflammation } \\
\hline $\mathrm{A} 1$ & $11(44.0 \%)$ & $4(16.0 \%)$ & & \\
\hline $\mathrm{A} 2$ & $14(56.0 \%)$ & $16(64.0 \%)$ & & \\
\hline \multirow[t]{2}{*}{ A3 } & $0(0.0 \%)$ & $5(20.0 \%)$ & & \\
\hline & $1.56 \pm 0.51$ & $2.04 \pm 0.61$ & & $0.006^{*}$ \\
\hline \multicolumn{5}{|l|}{ Stage of fibrosis } \\
\hline $\mathrm{F} 1$ & $12(48.0 \%)$ & $6(24.0 \%)$ & & \\
\hline F2 & $12(48.0 \%)$ & $12(48.0 \%)$ & & \\
\hline \multirow[t]{2}{*}{ F3 } & $1(4.0 \%)$ & $7(28.0 \%)$ & & \\
\hline & $1.56 \pm 0.58$ & $2.04 \pm 0.73$ & & $0.019 *$ \\
\hline
\end{tabular}

ALT - alanine aminotransferase, ALP - alkaline phosphatase, AST - aspartate aminotransferase, Hb - haemoglobin concentration, LS - liver stiffness, PPT - periportal tract thickening, PT - prothrombin time, PV - portal vein, SS - spleen stiffness, WBCS - white blood cells

Data were described using number and percent, in mean $\pm S D$

*statistically significant at $p \leqslant 0.05$

${ }^{k W} p$ - $p$ value for Kruskal-Wallis test, non-parametric test used to compare more than 2 groups

${ }^{F} p$ - $p$ value for ANOVA test, parametric test used to compare more than 2 groups 


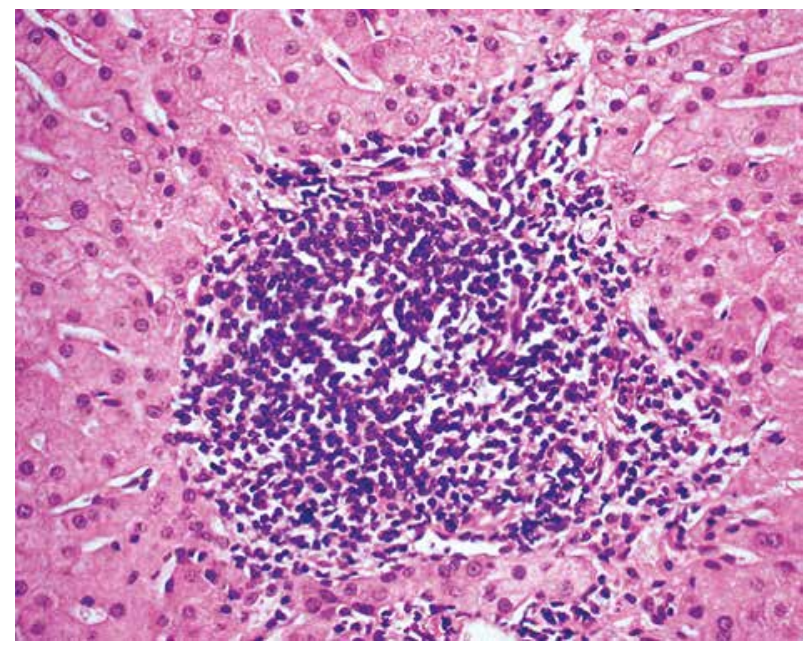

Fig. 2. Dense lymphocytic aggregation in a portal tract with chronic hepatitis $C$ patients

that measured in patients in group A $(2.85 \pm 0.70,2.46$ \pm 0.41 respectively and $p$-value $=0.021$ ).

\section{Correlation of liver and spleen stiffness assessed by ARFI with the stage of fibrosis}

Liver stiffness (ARFI L) in CHC (group A) patients who had stage 1 fibrosis (F1) ranged from 0.8 to $1.5 \mathrm{~m} / \mathrm{s}$ (mean $\pm \mathrm{SD}=1.10 \pm 0.21$ ) and in patients with significant fibrosis (F2 and F3) ARFI L ranged from 1.40 to $2.20 \mathrm{~m} / \mathrm{s}$ (mean $\pm \mathrm{SD}=1.82 \pm 0.24$ ). Taking a cut-off value of $>1.5 \mathrm{~m} / \mathrm{s}$ for differentiation between significant and insignificant fibrosis, it has a sensitivity of $92.31 \%$, a specificity of $100 \%$, an accuracy of $96 \%$ (pos-

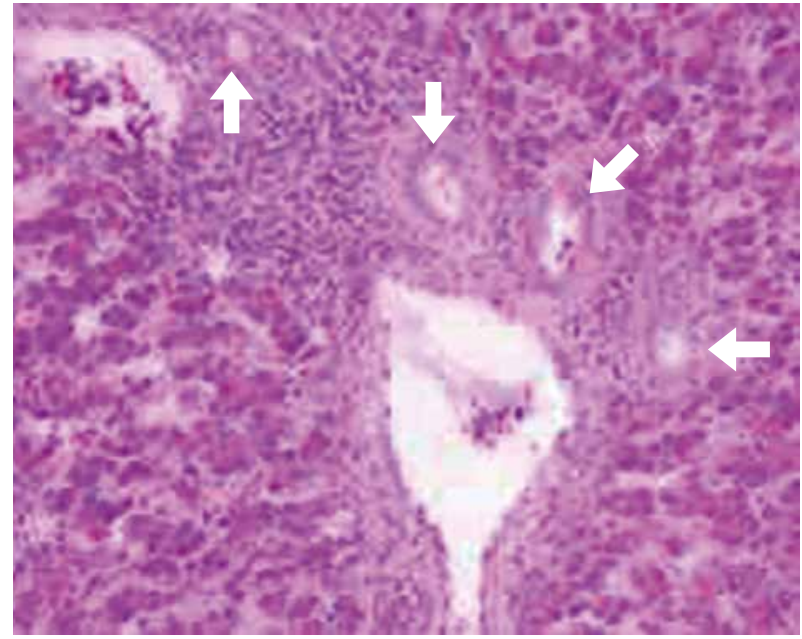

Fig. 3. Peri-portal fibrosis extending towards neighbouring portal triad and into the lobules (surrounded with arrows)

itive predictive value [PPV] of 100 , negative predictive value $[\mathrm{NPV}]$ of 92.3) and an area under the curve (AUC) of $0.99(p=0.001)$ (Fig. 4), while in group B, ARFI L ranged from 0.70 to $1.30 \mathrm{~m} / \mathrm{s}$ (mean $\pm \mathrm{SD}=$ $1.09 \pm 0.25$ ) in patients with F1. Meanwhile in patients with significant fibrosis, ARFI L ranged between 1.30 and $2.80 \mathrm{~m} / \mathrm{s}$ (mean $\pm \mathrm{SD}=1.99 \pm 0.53)$. The sensitivity was $84.21 \%$, the specificity was $100 \%$, the accuracy was $84 \%$ (PPV of 100, NPV of 66.6) and AUC of 0.96 $(p=0.001)$, when taking the cut-off point of $>1.3 \mathrm{~m} / \mathrm{s}$ (Fig. 5).

Group A patients had ARFI S of 1.70-2.82 m/s (mean $\pm \mathrm{SD}=2.17 \pm 0.36$ ) in $\mathrm{F} 1$ and $2.40-3.30 \mathrm{~m} / \mathrm{s}$ (mean $\pm \mathrm{SD}=2.72 \pm 0.26$ ) in patients with F2-F3), with

Table 2. Liver and spleen stiffness assessed by ARFI in the studied subjects

\begin{tabular}{|c|c|c|c|c|}
\hline & $\begin{array}{l}\text { Group A } \\
(n=25)\end{array}$ & $\begin{array}{l}\text { Group B } \\
(n=25)\end{array}$ & $\begin{array}{l}\text { Control } \\
(n=25)\end{array}$ & $P$ \\
\hline \multicolumn{5}{|l|}{ ARFI L (m/s) } \\
\hline Min.-Max. & $0.80-2.20$ & $0.70-2.80$ & $0.60-1.10$ & \multirow[t]{2}{*}{$<0.001^{*}$} \\
\hline Mean \pm SD & $1.48 \pm 0.43$ & $1.78 \pm 0.61$ & $0.83 \pm 0.16$ & \\
\hline$p_{1}$ & $<0.001^{*}$ & $<0.001^{*}$ & & \\
\hline$p_{2}$ & \multicolumn{2}{|c|}{0.062} & & \\
\hline \multicolumn{5}{|l|}{ ARFI $S(\mathrm{~m} / \mathrm{s})$} \\
\hline Min.-Max. & $1.70-3.30$ & $1.42-4.20$ & $1.30-2.20$ & \multirow[t]{2}{*}{$<0.001^{*}$} \\
\hline Mean $\pm S D$ & $2.46 \pm 0.41$ & $2.85 \pm 0.70$ & $1.75 \pm 0.23$ & \\
\hline$p_{1}$ & $<0.001^{*}$ & $<0.001^{*}$ & & \\
\hline$p_{2}$ & & & & \\
\hline
\end{tabular}

ARFI - acoustic radiation force impulse imaging, ARFI L - liver stiffness, ARFI S - splenic stiffness

$p-p$ value for F test (ANOVA) for comparing between the two studied groups

$p 1$ - $p$ value for post hoc test (Scheffe) for comparing between control and each other group

$p 2$ - $p$ value for post hoc test (Scheffe) for comparing between group $A$ and group $B$

*statistically significant at $p \leqslant 0.05$ 


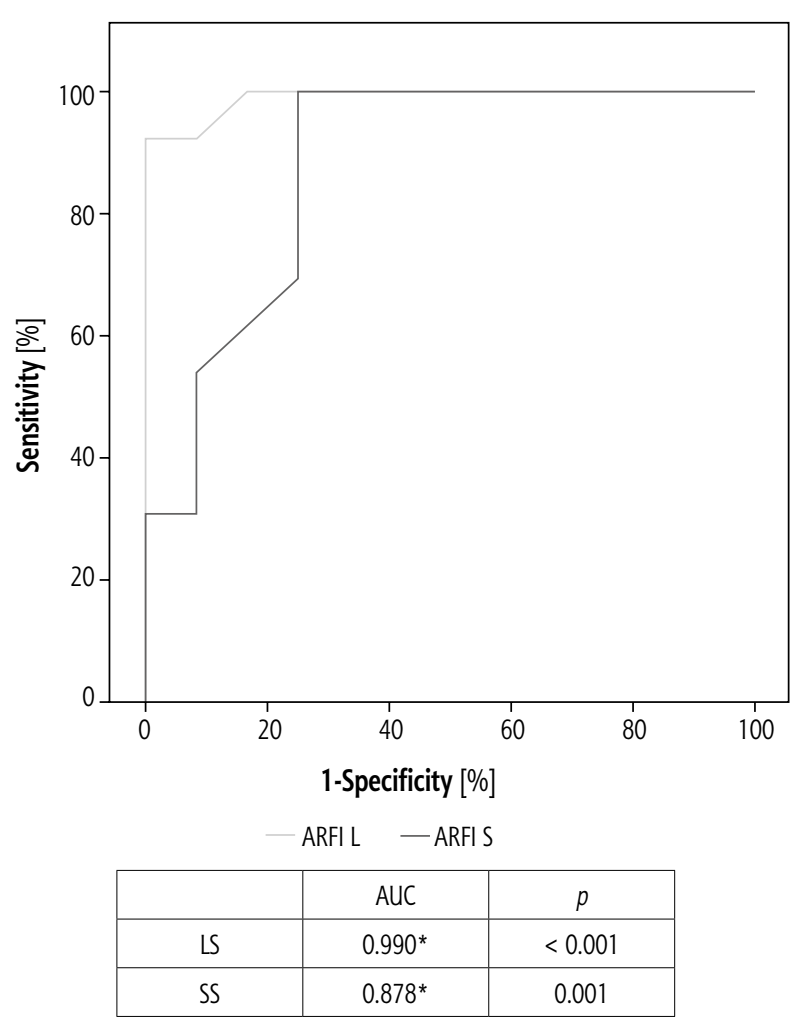

$A R F I L$ - acoustic radiation force impulse of the liver, ARFI S - acoustic radiation force impulse of the spleen, AUC - area under the curve

$p-p$ value for Mann-Whitney test for comparing between fibrosis categories *statistically significant at $p \leqslant 0.05$

Fig. 4. ROC curve for ARFI of group A

a sensitivity of $100 \%$, a specificity of $75 \%$ and an accuracy of $80 \%$ (PPV of 81.2 , NPV of 100) for a cutoff value of $>2.3 \mathrm{~m} / \mathrm{s}$ for differentiation between insignificant and significant fibrosis with AUC of 0.87 $(p=0.001)$ (Fig. 4). Meanwhile in group B, ARFI S ranged between 1.42 and $2.80 \mathrm{~m} / \mathrm{s}$ (mean $\pm \mathrm{SD}=2.07$ $\pm 0.46)$ and in patients with F2-F3 it ranged between 2.30 and $4.20 \mathrm{~m} / \mathrm{s}$ (mean $\pm \mathrm{SD}=3.10 \pm 0.57$ ), with a sensitivity of $94.74 \%$, a specificity of $83.33 \%$, an accuracy of 92\% (PPV of 94.7, NPV of 83.3) and AUC of 0.94 ( $p=0.001)$ for the same cut-off value (Fig. 5).

A remarkable correlation of ARFI $L$ with gender in both groups $\mathrm{A}$ and $\mathrm{B}$ existed, being stronger in males than females. Both ARFI L and S exhibited a correlation with age and shrunken liver as well.

\section{Discussion}

Clinical management of HCV patients encompasses non-invasive tests allowing staging of the liver disease and bypassing the liver biopsy. In the developing world, schistosomiasis is one of the most preva-

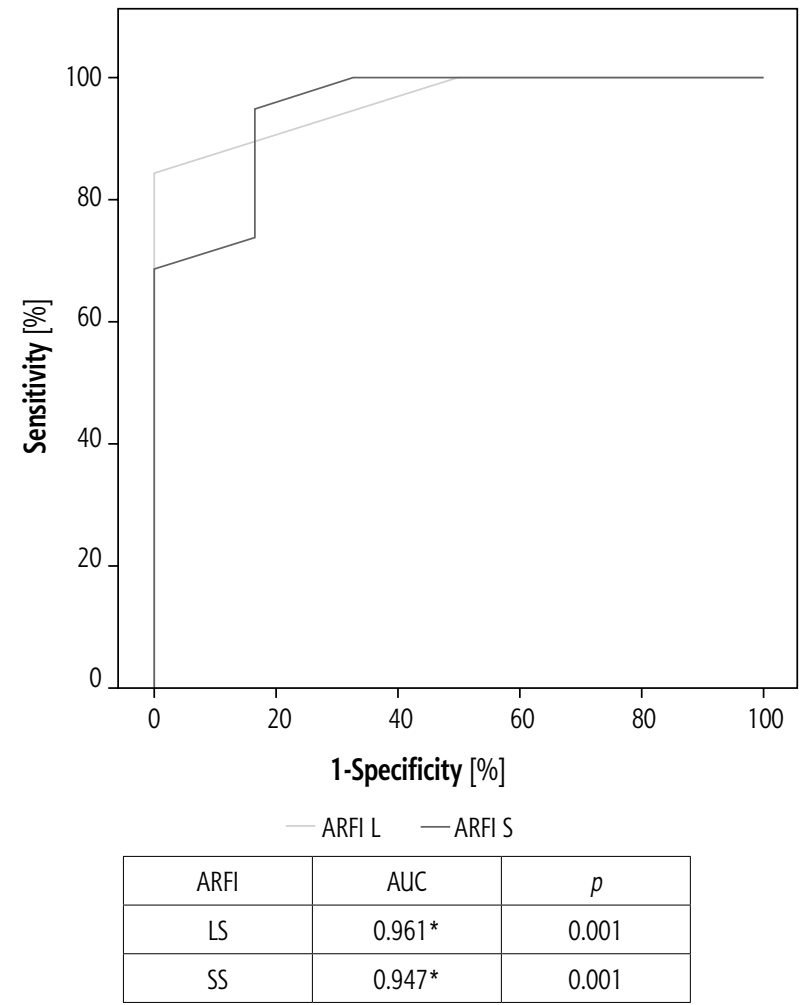

ARFI L - acoustic radiation force impulse of the liver, ARFIS - acoustic radiation force impulse of the spleen, $A U C$ - area under the curve

$p-p$ value for Mann-Whitney test for comparing between fibrosis categories *statistically significant at $p \leqslant 0.05$

Fig. 5. ROC curve for ARFI of group B

lent, though neglected, tropical infectious diseases. It is a major intravascular infection that has devastating public health consequences, thus vastly affecting the socioeconomic state [4-8]. We intended to assess the impact of a pre-existing schistosomiasis infection on ARFI L and S (as a reliable non-invasive approach for the staging of fibrosis in chronic HCV patients) in patients co-infected with HCV.

\section{Liver stiffness}

In a pooled meta-analysis consisting of 518 patients with chronic liver disease ( $83 \%$ with viral hepatitis) mean AUROCs of ARFI L was 0.87 for the diagnosis of significant fibrosis, and 0.93 for the diagnosis of liver cirrhosis [9]. In a meta-analysis of 36 studies (21 full paper publications and 15 abstracts) comprising 3951 patients mean AUROCs was 0.84 for the diagnosis of significant fibrosis, and 0.91 for the diagnosis of liver cirrhosis [10]. Cut-off values suggested in the two meta-analyses were $1.34-1.35 \mathrm{~m} / \mathrm{s}$ for the diagnosis of significant fibrosis and $1.80-1.87 \mathrm{~m} / \mathrm{s}$ for the diagnosis of cirrhosis. 
The study showed insignificantly higher values of liver stiffness assessed by ARFI in HCV patients co-infected with schistosomiasis than HCV mono-infected patients, assuming impaired performance of ARFI in the presence of co-infection, especially in F2 and F3 fibrosis stages.

This was further supported by Ramzy et al., who studied 352 cases, 122 of which were chronic HCV without antischistosomal antibody, 122 chronic HCV with positive antischistosomal antibodies and without PPT, and 108 chronic HCV with positive antischistosomal antibodies and an ultrasonographic picture of PPT. Although not statistically significant among the three groups, harmony existed between the histopathological (METAVIR) and the FibroScan results of the HCV patients (with no schistosomiasis infection). Despite the harmonization being higher among those with no PPT $(70.7 \%)$ and the discrepancy being higher among those with positive schistosomal serology (66.5\%), this relation was not statistically significant [11].

These study results are aligned with a previous one [12] in which 231 chronic HCV patients were enrolled. They were subdivided into a group with positive schistosomal serology and a second one with HCV patients having negative schistosomal serology. Liver stiffness measurements using FibroScan and reference needle-liver biopsy were attempted, concluding that the agreement between the FibroScan and the liver biopsy was more pronounced in HCV patients with negative schistosomal serology than in those with positive schistosomal serology. Another study confirmed that the disagreement between the results of liver biopsy and FibroScan was more obvious in those with positive schistosomal serology and HCV infection [13].

Furthermore, a cross-sectional study comprising 358 patients with schistosomiasis was conducted by Carvalho et al. [14] using point shear-wave elastography (pSWE). Patients were classified into two groups: 86 patients with mild PPT and 272 patients with significant PPT. The median pSWE of the significant fibrosis group was higher $(1.40 \mathrm{~m} / \mathrm{s})$ than that of the mild fibrosis group $(1.14 \mathrm{~m} / \mathrm{s}, p<0.001)$. AUC was 0.719 with $\leq 1.11 \mathrm{~m} / \mathrm{s}$ as the best cut-off value for excluding significant PPT. Sensitivity and negative predictive values were $80.5 \%$ and $40.5 \%$, respectively, whereas, for confirming significant PPT, the best cut-off value was $>1.39 \mathrm{~m} / \mathrm{s}$, with a specificity of $86.1 \%$ and a positive predictive value of $92.0 \%$.

There are intrinsic differences between men and women in the density of the extracellular matrix of the liver $[15,16]$. Our study reported that the mean ARFI L was significantly higher in males than in females. However, the influence of gender on LS is controver- sial. A large sample (923 healthy subjects) Italian study that used transient elastography (TE) revealed that the liver stiffness in males was significantly higher than in females [17]. A different study that used ARFI among 137 subjects found no statistically significant difference in mean ARFI values between males and females [18]. These discrepancies may be a result of different sample sizes, racial differences or differing imaging principles among TE and ARFI technologies.

\section{Spleen stiffness}

During the progression of liver cirrhosis and portal hypertension, passive congestion and tissue hyperplasia characterized by a combination of angiogenesis and fibrogenesis frequently occur in the spleen. All these changes result in increased spleen stiffness.

The current study demonstrated significantly higher values of spleen stiffness assessed by ARFI in patients co-infected with HCV and schistosomiasis than mono-infection with HCV, which was assumed to impair the performance of ARFI in presence of co-infection. This may be attributed to the higher number of patients with significant fibrosis (F2, F3) in group B (19 patients in group B vs. 13 in group A). Also, the mean value of the fibrosis stage was significantly higher in group B than A $(2.04 \pm 0.73,1.56 \pm 0.58$ respectively and $p=0.019$ ). Still, we cannot ignore the role of splenic congestion and tissue hyperplasia due to schistosomal infection as an explanation for these higher values of spleen stiffness in group B.

A study conducted by Veiga et al. [19] using transient elastography compared hepatosplenic schistosomiasis and HCV-cirrhotic patients; spleen stiffness values were comparable between schistosomiasis and cirrhotics. These discrepancies may be a result of different sample sizes, racial differences, and differing imaging principles among TE and ARFI technologies. Also, Veiga's study found no significant difference in splenic stiffness, as they included cirrhotic patients while we enrolled chronic hepatitis patients (lower fibrosis stage).

We have to emphasize that this study has several unique features; our study was conducted using ARFI, while most of the previous studies described in the literature were conducted using transient elastography. To our knowledge, this research is the first study of ARFI that has focused on Egyptian subjects and used ARFI technology to assess the spleen stiffness in an Egyptian patients with HCV mono-infection and coinfection with schistosomiasis. In future studies of chronic liver disease, our results may be used as a reference value for assessing the liver and spleen stiffness 
using ARFI in such groups of patients. The small sample size is the limitation of our study in addition to not correlating the tissue stiffness with the radiological staging of PPT.

\section{Conclusions}

The current study demonstrated insignificantly higher values of liver stiffness and significantly higher values of spleen stiffness assessed by ARFI in HCV patients co-infected with schistosomiasis than monoinfected patients.

\section{Disclosure}

The authors declare no conflict of interest.

\section{References}

1. Raghuwanshi B, Jain N, Jain M. Normal values in healthy liver in central India by acoustic radiation force impulse imaging. J Clin Diagn Res 2013; 7: 2498-2501.

2. Hotez PJ, Alvarado M, Basanez MG, et al. The Global Burden of Disease Study 2010: interpretation and implications for the neglected tropical diseases. PLoS Negl Trop Dis 2014; 8: e2865-e2873.

3. Gray DJ, Ross AG, Li YS, et al. Diagnosis and management of schistosomiasis. BMJ 2011; 342: d2651.

4. Chitsulo L, Engels D, Montresor A, et al. The global status of schistosomiasis and its control. Acta Trop 2000; 77: 41-51.

5. Engels D, Chitsulo L, Montresor A, et al. The global epidemiological situation of schistosomiasis and new approaches to control and research. Acta Trop 2002; 82: 139-146.

6. Steinmann P, Keiser J, Bos R, et al. Schistosomiasis and water resources development: systematic review, meta-analysis, and estimates of people at risk. Lancet Infect Dis 2006; 6: 411-425.

7. King CH, Dickman K, Tisch DJ. Reassessment of the cost of chronic helmintic infection: a meta-analysis of disability-related outcomes in endemic schistosomiasis. Lancet 2005; 365: 1561-1569.

8. WHO 2014. Schistosomiasis fact sheet 115. World Health Organization, Geneva, Switzerland.

9. Friedrich-Rust M, Nierhoff J, Lupsor M, et al. Performance of acoustic radiation force impulse imaging for the staging of liver fibrosis: a pooled meta-analysis. J Viral Hepat 2012; 19: e212-e219.

10. Nierhoff J, Chavez Ortiz AA, Herrmann E, et al. The efficiency of acoustic radiation force impulse imaging for the staging of liver fibrosis: a meta-analysis. Eur Radiol 2013; 23: 3040-3053.

11. Ramzy I, Elsharkawy A, Fouad R, et al. Impact of old Schistosomiasis infection on the use of transient elastography (Fibroscan) for staging of fibrosis in chronic HCV patients. Acta Tropica 2017; 176: 283-287.

12. Esmat G, Elsharkawy A, El Akel W, et al. Fibroscan of chronic HCV patients coinfected with schistosomiasis. Arab J Gastroenterol 2013; 14: 109-112.

13. Alboraie M, Khairy M, Elsharkawy M, et al. Value of Egy-Score in diagnosis of significant, advanced hepatic fibrosis and cirrhosis compared to aspartate aminotransferase-to-platelet ratio index, FIB-4 and Forns' index in chronic hepatitis $\mathrm{C}$ virus. Hepatol Res 2015; 45: 560-570.
14. Carvalho Santos J, Dória Batista A, Maria Mola Vasconcelos C, et al. Liver ultrasound elastography for the evaluation of periportal fibrosis in schistosomiasis mansoni: A cross-sectional study. PLoS Negl Trop Dis 2018; 12: 11.

15. Bissell DM. Sex and hepatic fibrosis. Hepatology 1999; 29: 988989.

16. Di Martino V, Lebray P, Myers RP, et al. Progression of liver fibrosis in women infected with hepatitis C: long-term benefit of estrogen exposure. Hepatology 2004; 40: 1426-1433.

17. Colombo S, Belloli L, Zaccanelli M, et al. Normal liver stiffness and its determinants in healthy blood donors. Dig Liver Dis 2011; 43: 231-236.

18. Madhok R, Tapasvi C, Prasad U, et al. Acoustic radiation force impulse imaging of the liver: Measurement of the normal mean values of the shearing wave velocity in a healthy liver. J Clin $\mathrm{Di}$ agn Res 2013; 7: 39-42.

19. Veiga ZST, Villela-Nogueira CA, Fernandes FF, et al. Transient elastography evaluation of hepatic and spleen stiffness in patients with hepatosplenic schistosomiasis. Eur J Gastroenterol Hepatol 2017; 29: 730-735. 